hep-th/9908077

TIFR-TH-99-41

August 1999

\title{
Discrete Flux as Quantum Hair
}

\author{
Atish Dabholkar And SANDiP P. TRIVEdi \\ Department of Theoretical Physics \\ Tata Institute of Fundamental Research \\ Homi Bhabha Road, Mumbai, India, 400005
}

\begin{abstract}
We investigate Yang-Mills theory on a spatial torus at finite temperature in the presence of discrete electric and magnetic fluxes using the AdS/CFT correspondence. We calculate the leading dependence of the partition function on the fluxes using the dual supergravity theory and comment upon the interpretation of these fluxes as discrete quantum hair for black holes in AdS spacetime.
\end{abstract}




\section{Introduction}

In this paper we calculate the finite temperature partition function of the Yang-Mills theory with sixteen supercharges on a torus as a function of discrete electric and magnetic fluxes. We perform this calculation in the large $N$ limit using the dual supergravity description in four and five dimensions [10, 1]. In this description, the thermal state of the gauge theory maps to a black hole [17] and we will show that the discrete flux in the gauge theory maps to a kind of quantum hair for the black hole. Thus, our calculation will reduce to determining how the partition function of a black hole depends on its quantum hair.

The study of discrete fluxes in nonabelian gauge theories was initiated by 't Hooft 15 following the analogies between confinement and superconductivity. Magnetic lines of force are expelled from the superconducting ground state and can penetrate the medium only in the form of thin flux tubes. Magnetic flux tubes exist as Nielsen-Olsen vortices and are locally stable. If a pair of magnetic monopole and antimonopole is introduced in the medium then the total flux between them is localized in a flux tube that connects the two charges giving rise to a linear confining potential between the two. Qualitatively, confinement in nonabelian gauge theories is the electric analog of superconductivity where the electric flux tubes are locally stable and can then be interpreted as QCD strings. To put this idea on a more quantitative basis, 't Hooft considered the gauge theory on a torus. This allows for the introduction of topological discrete fluxes in the pure gauge theory without quarks which are the analogues of electric and magnetic fluxes in the U(1) theory. By examining the dependence of the free energy on these fluxes, one can investigate the stability of the flux tubes to gain insight about the phases of the theory. In this paper we investigate some of these questions using the correspondence with supergravity which provides an explicit realization of these ideas.

By now a fair amount is known about the finite temperature SYM theory we will consider here. For example, the spatial Wilson loop has been calculated in the theory and shows area law behavior thereby indicating that the theory has locally stable flux tubes [11, 13]. In this paper we will follow the general framework discussed in [2, [8] for including discrete flux in supergravity. Our calculation will be done in an approximation where the string tension of these flux tubes is much bigger than the size of the torus. As we will see, in this approximation, the dominant configurations contributing to the partition function have a simple interpretation in the gauge theory: they correspond to the worldsheets of flux tubes wrapping appropriate two cycles of the torus. We will do the calculation in both the four-dimensional and the five-dimensional theory. The latter, at energies low compared to the temperature, should flow to the four-dimensional pure glue gauge theory without supersymmetry. Unfortunately, as is well known, in the supergravity regime we use here, there are extra Kaluza-Klein states in the theory which do not decouple [17, 0]. Thus, from the point of view of the non-supersymmetric gauge theory, our calculation is at best an approximation, somewhat analogous to the strong coupling expansion in lattice gauge theory. In fact, as we will show, the dual supergravity yields an answer which, in its important quantitative features, agrees with the strong coupling expansion. These features are in 
accord with the expectation about confining theories and are likely to be universal.

It is also useful to consider the discussion in this paper from the perspective of the supergravity theory. As was mentioned above, a black hole in the bulk is represented as a thermal state in the boundary gauge theory. The classical no-hair theorems of black hole physics then turn into a familiar statement in the gauge theory: a thermal state is only characterized by its mass, angular momentum, and various conserved global charges. But as is well known [3, 5], black holes can also possess quantum hair. One might wonder what their description is in the boundary theory. For the quantum hair considered in this paper they turn out to be related to the the discrete electric and magnetic fluxes of the gauge theory.

This paper is organized as follows. The four-dimensional theory with $N=4$ supersymmetric is discussed in $\S 2$. The AdS/CFT correspondence for the blackhole geometry is reviewed in $\S 2.1$, discrete fluxes from the gauge theory and supergravity points of view are described in $\S 2.2$ and $\S 2.3$ respectively, and the calculation of the partition function is discussed in $\S 2.4$. Nonsupersymmetric gauge theories in three and four dimensions are discussed in $\S 3$ and $\S 4$ respectively starting with a supersymmetric theory in one extra dimension compactified on a circle with thermal boundary conditions that break supersymmetry.

\section{Discrete Fluxes in the AdS/CFT Correspondence}

\subsection{The Black Hole Geometry}

In this section we consider the $N=4$ supersymmetric $S U(N)$ Yang-Mills theory on a spatial torus $\mathbf{T}^{\mathbf{3}}$ at finite temperature $T$. The boundary spacetime $\mathbf{M}$ in this case has topology $\mathbf{T}^{\mathbf{3}} \times \mathbf{S}^{\mathbf{1}}$. By the AdS/CFT correspondence [10, 17], this boundary theory is dual to a bulk supergravity theory on $\mathbf{B} \times \mathbf{S}^{\mathbf{5}}$ where $\mathbf{B}$ is an Einstein manifold that has $\mathbf{M}$ as the boundary at infinity. The relevant manifold $\mathbf{B}$ is well studied and corresponds to a Euclidean Schwarzschild black hole in AdS space [8]. In Poincare coordinates, the metric is:

$$
d s^{2}=\left(\frac{r^{2}}{R^{2}}-\frac{c G_{5} M}{r^{2}}\right) d x_{0}^{2}+\frac{d r^{2}}{\left(\frac{r^{2}}{R^{2}}-\frac{c G_{5} M}{r^{2}}\right)}+\frac{r^{2}}{R^{2}}\left(d x_{1}^{2}+d x_{2}^{2}+d x_{3}^{2}\right),
$$

where $R=\left(4 \pi g_{s} \alpha^{\prime} N\right)^{1 / 4}$ is the radius of curvature of AdS space, $G_{5}=G_{10} /\left(R^{5} \pi^{3}\right)$ is the five-dimensional Newton's constant, $M$ is the mass of the black hole, and $c=\frac{32}{3 \pi}$. The black hole horizon is at

$$
r_{H}=\left(c G_{5} M R^{2}\right)^{1 / 4}
$$

and the temperature is

$$
T=\frac{1}{\pi} r_{H} / R^{2}
$$

${ }^{*}$ Since we are working in a situation where the spatial boundary is $\mathbf{T}^{3}$ as opposed to $\mathbf{S}^{3}$, the relevant solution is obtained by taking the large mass limit of the metric in [17]. 
The coordinates $x_{i}(i=1,2,3)$ parametrize a 3 -torus. For simplicity we consider a cubic torus of size $L$ so that $x_{i}$ are identified with $x_{i}+L$. Note that the physical size of the torus at the radial position $r$ in the bulk is $r L / R$. In the black hole geometry, $r$ is bounded from below by the radius of black hole horizon $r_{H}$. As long as $r_{H} L / R$ is large compared to the string scale, we expect that the dual supergravity description will be well-defined. By contrast, at zero temperature ( $M=0$ in the above formulae), the size of the torus becomes vanishingly small as $r$ goes to zero. The supergravity description then becomes inadequate at small $r$ because the string modes that wind around the torus become massless near $r=0$ and have to be included in the low energy description.

\subsection{Fluxes in the Boundary Gauge Theory}

The finite temperature partition function of the Yang-Mills theory on the boundary is obtained by computing the Euclidean path integral on the 4 -torus $\mathbf{T}^{\mathbf{3}} \times \mathbf{S}^{\mathbf{1}}$. In the $N=4$ supersymmetric theory, the center $\mathbf{Z}_{N}$ of $S U(N)$ acts trivially because all fields transform in the adjoint representation. Therefore, one is really dealing with an $S U(N) / \mathbf{Z}_{N}$ gauge bundle on a 4-torus. Such gauge bundles are labeled by six topological invariants $n_{\mu \nu}=-n_{\nu \mu}$ $(\mu, \nu=0, \ldots, 3)$ which are all integers modulo $N$. We would like to calculate the dependence of the partition function on these integers.

Let us briefly review the origin of these integers and their physical interpretation [15, 12]. In the path integral, one sums over field configurations that are periodic up to a gauge transformationf. This allows for twisted gauge fields. For example, in the $x_{1}-x_{2}$ plane with $x_{0}$ and $x_{3}$ fixed, a field $\Phi$ satisfies the boundary condition

$$
\begin{aligned}
& \Phi\left(L, x_{2}\right)=\Omega_{1}\left(x_{2}\right) \Phi\left(0, x_{2}\right) \\
& \Phi\left(x_{1}, L\right)=\Omega_{2}\left(x_{1}\right) \Phi\left(x_{1}, 0\right) .
\end{aligned}
$$

Here $\Omega \Phi$ denotes schematically the appropriate gauge transformation of $\Phi$ by $\Omega: A_{\mu} \rightarrow$ $\Omega A_{\mu} \Omega^{-1}-i \partial_{\mu} \Omega \Omega^{-1}$ for the gauge fields, $\lambda \rightarrow \Omega \lambda \Omega^{-1}$ for the gauginoes, and similarly for the scalars. For consistency, the gauge transformations must satisfy the cocycle condition

$$
\begin{aligned}
\Phi(L, L) & =\Omega_{1}(L) \Phi(0, L)=\Omega_{1}(L) \Omega_{2}(0) \Phi(0,0) \\
& =\Omega_{2}(L) \Phi(L, 0)=\Omega_{2}(L) \Omega_{1}(0) \Phi(0,0) .
\end{aligned}
$$

Therefore, $\Omega_{1}(L) \Omega_{2}(0)$ and $\Omega_{2}(L) \Omega_{1}(0)$ must be equal up to an element of the center $\mathbf{Z}_{N}$,

$$
\Omega_{1}(L) \Omega_{2}(0)=\exp \left(\frac{2 \pi i n_{12}}{N}\right) \Omega_{2}(L) \Omega_{1}(0) .
$$

The integer $n_{12}$ is defined modulo $N$ and is a topological invariant because it cannot be changed by a periodic gauge transformation or by smooth deformations of the field. There

\footnotetext{
$\dagger$ The fermions are actually periodic on $\mathbf{T}^{\mathbf{3}}$ and antiperiodic on $\mathbf{S}^{\mathbf{1}}$ but we will ignore this distinction in this subsection
} 
are altogether six independent integers $n_{\mu \nu}$ corresponding to the six 2-cycles of the 4-torus that completely specify the topological class of the gauge fields.

The integers $\left\{n_{i j}\right\}(i, j=1,2,3)$ are related to the magnetic flux on the torus. For example, consider the contractible Wilson loop $\Omega_{1}(L) \Omega_{2}(0) \Omega_{1}^{-1}(0) \Omega_{2}^{-1}(L)$ that measures the total magnetic flux passing through the $x_{1}-x_{2}$ plane. We immediately see from the cocycle condition eq.(6) that there is $n_{12}$ units of magnetic flux in the $x_{3}$ direction for the twisted gauge fields considered above. In general, one can define the integer $m_{i} \equiv \frac{1}{2} \epsilon_{i j k} n_{j k}$ as the magnetic flux in the $i$ th direction.

The physical interpretation of $n_{0 i}$ is clearest in the Hamiltonian formalism. In the gauge $A_{0}=0$, the theory has a residual invariance under time-independent gauge transformations. States in the physical Hilbert space must furnish a representation of this invariance group. For a gauge transformation $\Omega(\mathbf{x})$ that is continuously connected to the identity, one must choose the trivial representation for the corresponding operator consistent with Gauss Law:

$$
\hat{\Omega}(\mathbf{x})|\psi\rangle=|\psi\rangle
$$

However, for the $S U(N) / \mathbf{Z}_{N}$ theory on $\mathbf{T}^{\mathbf{3}}$ there are quasiperiodic gauge transformations that are is not continuously connected to the identity. A quasiperiodic gauge transformation is periodic modulo an element of the center. For example, consider a gauge transformation that is quasiperiodic in the $x_{3}$ direction

$$
\Omega\left[k_{3}\right]\left(x_{1}, x_{2}, L\right)=\Omega\left[k_{3}\right]\left(x_{1}, x_{2}, 0\right) \exp \left(\frac{2 \pi i k_{3}}{N}\right)
$$

and periodic in the $x_{1}, x_{2}$ directions. Two such gauge transformations that are labeled by the same integer $k_{3}$ have the same action on physical states because they differ by a homotopically trivial gauge transformation that leaves such states invariant as in eq.(7). Such gauge transformations obviously generate a $\mathbf{Z}_{N}$ group and moreover commute with the Hamiltonian. One can choose the physical states to be eigenstates of this gauge transformation

$$
\hat{\Omega}\left[k_{3}\right]|\psi\rangle=\exp \left(\frac{2 \pi i k_{3} e_{3}}{N}\right)|\psi\rangle
$$

for some integer $e_{3}$ modulo $N$. To see that the integer $e_{3}$ can be interpreted as the electric flux in the $x_{3}$ direction, consider the action of $\Omega\left[k_{3}\right]$ on a Wilson loop

$$
\mathcal{A}\left(C_{3}\right)=\operatorname{tr} P \exp \left(i \int_{C_{3}} d x^{\mu} A_{\mu}\right)
$$

that runs along a curve $C_{3}$ in the $x_{3}$ direction at $x_{1}=x_{2}=0$. It is clear that

$$
\hat{\Omega}\left[k_{3}\right] \mathcal{A}\left(C_{3}\right)=\mathcal{A}\left(C_{3}\right) \hat{\Omega}\left[k_{3}\right] \exp \left(\frac{2 \pi i k_{3}}{N}\right) .
$$

Thus $\mathcal{A}\left(C_{3}\right)$ acting on a state increases the value of $e_{3}$ by one unit. Since the Wilson loop creates a line of electric flux, it is natural to regard $e_{3}$ as the discrete electric flux. 
One can project onto states with a well defined electric flux $e_{3}$ by using the projection operator

$$
\mathcal{P}\left(e_{3}\right)=\frac{1}{N} \sum_{k_{3}} \exp \left(\frac{-2 \pi i k_{3} e_{3}}{N}\right) \hat{\Omega}\left[k_{3}\right] .
$$

The finite temperature partition sum over states with a specified value of $e_{3}$ is then given by

$$
Z\left(e_{3}\right) \equiv \operatorname{Tr}\left[\mathcal{P}\left(e_{3}\right) \exp (-H / T)\right]=\frac{1}{N} \sum_{k_{3}} \exp \left(\frac{-2 \pi i k_{3} e_{3}}{N}\right) \operatorname{Tr}\left[\hat{\Omega}\left[k_{3}\right] \exp (-H / T)\right]
$$

It is easy to see that each term $\operatorname{Tr}\left[\hat{\Omega}\left[k_{3}\right] \exp (-H / T)\right]$ in the sum can be expressed as a Euclidean functional integral over gauge fields that are twisted in the $x_{0}-x_{3}$ plane as in eq.(四) with $n_{03}=k_{3}$. The partition function $Z\left(e_{3}\right)$ is then obtained as a discrete Fourier transform using eq.113). Thus, the integer $k_{3}$ is conjugate to the electric flux $e_{3}$.

More generally, the electric fluxes $\left\{e_{i}\right\}$ are the quantum numbers of the $\mathbf{Z}_{N}^{3}$ electric symmetry and are conjugate to the integers $\left\{k_{i} \equiv n_{0 i}\right\}$. In addition, we also have $\mathbf{Z}_{N}^{3}$ magnetic symmetry. The integers $m_{i}$ that we defined earlier can equivalently be regarded as the quantum numbers of the this magnetic symmetry in the dual description.

\subsection{Fluxes in the Bulk Supergravity}

We would like to identify the topological $\mathbf{Z}_{N}^{6}$ symmetry and the quantum numbers that correspond to the discrete electric and magnetic fluxes in the dual supergravity theory. We will follow the general framework described in [18, 2] although the specific context here is somewhat different.

Consider, for example, the electric flux $e_{3}$ which is the quantum number of the $\mathbf{Z}_{N}$ symmetry that is generated by $\hat{\Omega}\left[k_{3}\right]$. A defining property of $\hat{\Omega}\left[k_{3}\right]$ is the commutation relation eq.(11) with the Wilson loop $\mathcal{A}\left(C_{3}\right)$. In the AdS/CFT correspondence, a Wilson loop $\mathcal{A}(C)$ that runs along a curve $C$ in the Yang-Mills theory is identified with the boundary of a fundamental string worldsheet $D$ that extends in the bulk geometry [11, 13]. For $\mathcal{A}\left(C_{3}\right)$, the corresponding string worldsheet $D_{3 r}$ extends, at a fixed Euclidean time $x_{0}$, in the $x_{3}-r$ plane such that $\partial D_{3 r}=C_{3}$. The worldsheet couples to the NS-NS 2-form field $B^{N S}$ through the coupling

$$
\exp \left(i \int_{D_{3 r}} B^{N S}\right)
$$

In particular, if we have a nonzero expectation value

$$
a=\int_{D_{3 r}} B^{N S}
$$

in the bulk then the worldsheet would pick up a phase $e^{i a}$. It follows from the commutation relations eq.(11) that the operator that corresponds to $\hat{\Omega}\left[k_{3}\right]$ would shift this expectation value

$$
a \rightarrow a+2 \pi k_{3} / N
$$


We will see that in the supergravity theory, there is a natural candidate for this $\mathbf{Z}_{N}$ shift symmetry. Obviously, the generator of this symmetry would be the momentum conjugate to $a$ which we can find from the classical action and the corresponding translation operator can then be identified with $\hat{\Omega}\left[k_{3}\right]$.

The relevant part of the supergravity Lagrangian on the Euclidean AdS space is

$$
\mathcal{L}=\frac{1}{2 g_{s}^{2}}\left(\left|d B^{N S}\right|^{2}+\left|d B^{R}\right|^{2}\right)+\frac{i N}{4 \pi}\left(B^{N S} \wedge d B^{R}-B^{R} \wedge d B^{N S}\right) .
$$

The first two terms are the usual kinetic terms restricted to the AdS part. The last two terms are topological and arise from the Chern-Simons coupling in ten dimensions of the two B-fields to the 5 -form field strength $G_{5}$. The factor of $N$ arises from the integration of $G_{5}$ over the sphere $\mathbf{S}^{5}$ and corresponds to the total 5 -form flux of $N$ D3-branes.

Since we are interested in spatially constant modes of the $B$ fields, we can integrate over space for the relevant modes and reduce this problem to particle mechanics. Let us define

$$
b=\int_{\Sigma_{12}} B^{R}
$$

for a nontrivial cycle $\Sigma_{12}$ along $x_{1}-x_{2}$ directions. Then the Lagrangian after an integratiioin by parts reduces to

$$
L=\frac{1}{2}\left(\mu_{1} \dot{a}^{2}+\mu_{2} \dot{b}^{2}\right)+\frac{i N}{2 \pi} a \dot{b} .
$$

Here the dot indicates a derivative with respect to the Euclidean time. The regularized effective masses $\mu_{1}, \mu_{2}$ arise from integrating the $r$ dependence of the metric in the original Lagrangian but their precise form will not be important in what follows. This is the Lagrangian of a particle moving on a plane in the presence of a constant magnetic field of magnitude $N / 2 \pi$ perpendicular to the plane. We also know that the 2 -form $B$ fields are both compact gauge fields because both the D-string and the F-string charge is quantized. Consequently, their zero modes $a$ and $b$ are compact coordinates with period $2 \pi$.

Now, without the coupling to the magnetic field, the Lagrangian clearly has a $U(1)$ shift symmetry $a \rightarrow a+c$ for an arbitrary periodic constant $c$. The coupling to the magnetic field breaks this $U(1)$ to a $\mathbf{Z}_{N}$ subgroup. This follows from the the fact that $e^{-\int L}$ would be invariant under the shift of $a \rightarrow a+2 \pi / N$ because $b$ has period $2 \pi$. It will suffice for our purposes to consider the limit of large magnetic field and drop the kinetic terms. The remaining Lagrangian is automatically first order and we can readily identify $b$ as the momentum conjugate to $a$. They would satisfy the Heisenberg commutation relation

$$
[\hat{a}, \hat{b}]=2 \pi i / N \text {. }
$$

Using this commutator it then follows that in our original problem we have the operator relation

$$
\exp \left(i k_{3} \int_{\Sigma_{12}} \hat{B}^{R}\right) \exp \left(i \int_{D_{3 r}} \hat{B}^{N S}\right)=\exp \left(i k_{3} \int_{\Sigma_{12}} \hat{B}^{R}\right) \exp \left(i \int_{D_{3 r}} \hat{B}^{N S}\right) \exp \left(\frac{2 \pi i k_{3}}{N}\right)
$$


which is identical to the relation eq.(11) that we were seeking. It implies the operator identification

$$
\hat{\Omega}\left[k_{3}\right] \leftrightarrow \exp \left(i k_{3} \int_{\Sigma_{12}} \hat{B}^{R}\right)
$$

A state in supergravity with a well defined flux $e_{3}$ is an eigenstate of this operator with

$$
\int_{\Sigma_{12}} B^{R}=\frac{2 \pi e_{3}}{N}
$$

By an analogous reasoning one can see that turning on $m_{3}$ units of magnetic flux in the $x_{3}$ direction then corresponds to turning on an expectation value

$$
\int_{\Sigma_{12}} B^{N S}=\frac{2 \pi m_{3}}{N}
$$

This also follows from the $S L(2, \mathbf{Z})$ duality symmetry which in the Type-IIB theory interchanges $B^{R}$ and $B^{N S}$ and in the Yang-Mills theory on the D3-brane worldvolume exchanges electric and magnetic fluxes. Under this duality the Wilson loop $\mathcal{A}(C)$ that creates electric flux tube gets interchanged with the 't Hooft loop $\mathcal{B}(C)$ which creates a magnetic flux tube. In the supergravity theory, $\mathcal{B}(C)$ is thus a boundary of a D-string worldsheet. In general, turning on the fluxes $e_{i}$ and $m_{i}$ in the Yang-Mills theory corresponds in the supergravity to turning on the expectation values

$$
\int_{\Sigma_{i j}} B^{R}=\frac{2 \pi \epsilon_{i j k} e_{k}}{N}, \quad \int_{\Sigma_{i j}} B^{N S}=\frac{2 \pi \epsilon_{i j k} m_{k}}{N} .
$$

\subsection{Finite Temperature Partition Function}

Having identified the discrete fluxes in the supergravity description we now turn to calculating the finite temperature partition function. When the 2 -form gauge potentials are zero, we saw in section 2.1 that the relevant configuration in the supergravity theory is a black hole. What happens when the gauge potentials are turned on? A careful quantization of the zero modes as in [2] shows that, when the gauge potentials are constant and take the quantized values, there is no extra cost in energy. In turn this means that the metric, the 5 -form field strength, and the dilaton stay unchanged from their values in the black hole background. Thus the discrete fluxes do not change the classical behavior of the black hole and the related thermodynamic quantities.

Once quantum fluctuations around the black holes background are included though a dependence on the fluxes does arise. These quantum fluctuations include Euclidean fundamental string worldsheets that wrap around non-trivial 2-cycles in the bulk geometry. These can be viewed as F-string instantons and in this instanton sector the partition function depends on the expectation value of the 2 -form gauge potential $B^{N S}$. One can also have D-string instantons that are sensitive to $B^{R}$ and more generally $(p, q)$-string instantons [14, 19. The discrete fluxes thus manifest themselves in the dual supergravity theory as quantum hair [3, 5]. 
Let us first consider the case where a single component of magnetic flux $m_{3}$ is turned on. From eq.(24), we see that the quantum fluctuations of relevance then will involve fundamental string Euclidean worldsheets that wrap around $\Sigma_{12}$. We can organize the partition function as a sum over configurations that wrap $n$ times around the 2-cycles:

$$
Z=Z_{\text {sugra }}+\sum_{n} Z_{(\text {sugra }+ \text { nstrings })}
$$

The leading term $Z_{\text {sugra }}$ is the contribution of the pure supergravity theory without any extra strings. It is determined by the black hole solution, eq.(四), to be :

$$
Z_{\text {sugra }}=e^{\left(c_{2} N^{2} T^{3} L^{3}\right)}
$$

where $c_{2}=\frac{\pi^{2}}{8}$. To precisely calculate the next term $Z_{(\text {sugra }+1 \text { string })}$ we would really need to know Type-IIB string theory on the black hole background or at least the sigma model in the Green-Schwarz formalism. We will estimate here the cost of including the string instanton using the Nambu-Goto action:

$$
Z_{(\text {sugra }+1 \text { string })}=e^{\left(c_{2} N^{2} T^{3} L^{3}\right)} \int D X e^{-S_{N G}+i \int B^{N S}} .
$$

The dominant contribution that is sensitive to the magnetic flux eq.(24) is obtained by the minimum area surface $\Sigma_{12}$ that extremizes the Nambu-Goto action. From eq.(1) we see that this surface arises from a string world sheet located at the horizon in the radial direction which spans the $x_{1}-x_{2}$ plane at some definite value of $x_{3}$.

The area of this minimal surface in units of the fundamental string tension is,

$$
A_{H} /\left(2 \pi \alpha^{\prime}\right)=\frac{1}{2 \pi} \frac{r_{H}^{2} L^{2}}{R^{2} \alpha^{\prime}} .
$$

Including the effects of an anti-string (or equivalently a string oppositely wound in the $x_{1}-x_{2}$ plane) gives:

$$
Z_{(\text {sugra }+1 \text { string })}=e^{\left(c_{2} N^{2} T^{3} L^{3}\right)} 2 C_{F} e^{-A_{H} /\left(2 \pi \alpha^{\prime}\right)} \cos \left(\frac{2 \pi m_{3}}{N}\right) .
$$

The coefficient $C_{F}$ arises in the string path integral from fluctuations around the minimal area surface. It includes a contribution, $L T$, due to the zero mode associated with the location of the surface in the $x_{3}$ direction, and the contribution due to non-zero modes as well. We also need to include the fluctuation of the fermionic modes. In particular, we need to worry if there are fermionic zero modes that might make the contribution vanish as a consequence of spacetime supersymmetry. We expect however that the zero modes of the spacetime Green Schwarz fermions will be lifted because the thermal boundary condition breaks supersymmetry completely.

Using eq.(3), eq.(29), the minimum area can be re-expressed as:

$$
e^{-A_{H} /\left(2 \pi \alpha^{\prime}\right)}=e^{-\pi^{3 / 2} \sqrt{g_{s} N} T^{2} L^{2}}
$$


Not surprisingly the coefficient $\pi^{3 / 2} \sqrt{g_{s} N} T^{2}$ is identical to the string tension of the electric flux tube in the finite temperature $N=4$ theory. From the supergravity point of view, the theory has stable flux tubes because at finite temperature the classical geometry is given by the black hole geometry, eq(1), in which the value of the metric parallel to the brane goes to a minimum non-zero value at the horizon [17, 4]. Thus the behavior of large Wilson loops is determined by the geometry at the horizon which also determines the exponential factor, eq.(31), above.

The contributions to the partition function from the single string instanton in the case of a general electric and magnetic flux can now be written down by including the contributions of $\mathrm{F}$ and $\mathrm{D}$ strings wrapping the corresponding two-cycle:

$$
\begin{aligned}
Z_{(\text {sugra }+1 \text { string })}\left[m_{j}, e_{i}\right]= & e^{\left(c_{2} N^{2} T^{3} L^{3}\right)}\left[2 C_{F} e^{-\pi^{3 / 2} \sqrt{g_{s} N} T^{2} L^{2}} \sum_{j} \cos \left(\frac{2 \pi m_{j}}{N}\right)\right. \\
& \left.+2 C_{D} e^{-\pi^{3 / 2} \sqrt{N / g_{s}} T^{2} L^{2}} \sum_{i} \cos \left(\frac{2 \pi e_{i}}{N}\right)\right]
\end{aligned}
$$

There are subleading contributions to eq.(32) which arise from string-worldsheets that either wrap multiply or wrap more than one two-cycle simultaneously. However, these contributions are exponentially suppressed compared to those included in eq.(32) as long as

$$
\sqrt{N g_{s}} T^{2} L^{2} \gg 1, \quad \sqrt{N / g_{s}} T^{2} L^{2} \gg 1
$$

For small $g_{s}$ the first condition is more restrictive. Physically, eq.(33) means that the torus has a size much bigger than the electric and magnetic string tensions. When this is true the contributions to the partition function from multi-string sectors are also suppressed. In addition, there are subleading contributions from $(p, q)$ string worldsheets which we discuss shortly in eq.(34). Eq.(32) thus gives the leading dependence of the partition function on the discrete fluxes. It is the main result of this section.

Some comments are now in order. First, it is worth noting that the minimum area surface which gave the dominant contribution to eq.(32) above has a simple interpretation in the gauge theory as well. It corresponds to an electric or magnetic flux tube, which is small at first but grows with time to span the full 2-cycle. Second, we have neglected terms which are down in the $\alpha^{\prime}$ and $g_{s}$ expansions here. Thus our result pertains to the gauge theory at large $\mathrm{N}$ and strong 't Hooft coupling. Third, the result above does not apply to the zero temperature case. In this limit the condition, eq.(33), is no longer met and the contributions from multiply-wound and multi-string states cannot be neglected.

Finally, to understand the $S L(2, \mathbf{Z})$ transformation properties of eq.(32) one needs to include the effects of general $(p, q)$ strings as well. Doing so gives a partition function:

$$
Z\left[m_{i}, e_{j}\right](\tau)=\sum_{i, j} \sum_{p, q} C_{p, q}(\tau) \exp \left(-A T_{p, q}(\tau)\right) \cos \left[\frac{2 \pi}{N}(-p \cdot m+q \cdot e)\right] .
$$

Here, $\tau=\chi+i e^{-\phi}$ is the axion-dilaton field and $C_{p, q}(\tau)$ is the determinant of small fluctuations which can in general depend on $\tau$. The string tension $T_{p, q}(\tau)$ of the $(p, q)$ string and 
the area of the two-cycle $A=2 \pi^{5 / 2} \alpha^{\prime} \sqrt{N} T^{2} L^{2}$ are both measured in the Einstein metric. Both $\left(e_{i}, m_{i}\right)$ and $\left(p_{i}, q_{j}\right)$ transform as vectors under the $S L(2, \mathbf{Z})$ transformation, and $\tau$ transforms as usual by fractional linear transformation. Keeping this in mind we see that the last two terms of eq.(34) are invariant. Determining the full transformation properties of $Z\left[m_{i}, e_{j}\right]$ requires additional information about $C_{p, q}$ though which is beyond the scope of this paper.

\section{Non-Supersymmetric Gauge Theory in Three dimensions}

At energies smaller than the temperature, the four dimensional theory reduces to a nonsupersymmetric theory in three dimensions. The fermions and scalars of the $N=4$ theory acquire temperature dependent masses leaving the pure glue degrees of freedom at low energies. The results of the previous section can be reinterpreted to tell us about the behavior of this theory. Before proceeding though it is important to emphasize that the resulting theory is quite different from the usual definition of three-dimensional QCD. From the threedimensional point of view the temperature acts like a cutoff. We mentioned in the previous section that the confining scale in the theory is of order $\left(g_{s} N\right)^{1 / 4} T$. Thus, in the limit of strong 't Hooft coupling considered here, the theory is already strongly coupled at the cutoff scale. Despite these differences it is interesting to ask how the three-dimensional theory behaves as a function of the discrete fluxes. The resulting answers are analogous to those obtained in the strong coupling expansion of lattice gauge theory which, despite differences with the continuum theory, are often illuminating.

We denote the two spatial directions of the three-dimensional theory as $x_{1}, x_{2}$ and the time direction as $x_{3}$. There is one integer $m_{3}$ which characterizes the magnetic flux in the $x_{1}-x_{2}$ plane. There are two electric fluxes in the $x_{1}, x_{2}$ directions respectively, characterized by the integers, $e_{1}, e_{2}$. We are interested in the partition function of this theory when both the spatial directions and the time direction are taken to be circles of length $L$, and periodic boundary conditions are imposed in all three directions. As was discussed in $\S 2$ the dependence on the electric flux $e_{i}$ is related by a Fourier transform to the partition function obtained by introducing twisted boundary conditions in the $x_{1}-x_{3}$ and $x_{2}-x_{3}$ directions.

In the supergravity theory the background geometry describing the theory is of course still described by the black hole, eq.(11). However, in the three-dimensional setting the coordinate axis have a somewhat different interpretation. $x_{0}$ can be regarded as the extra spatial direction along which supersymmetry breaking boundary conditions are imposed, while $x_{3}$ is the Euclidean continuation of the time direction.

The leading dependence of the partition function on the fluxes is therefore obtained by keeping the first term in eq. (32) above:

$$
\begin{aligned}
Z\left[m_{3}, n_{1}, n_{2}\right]= & e^{\left(c_{2} N^{2} T^{3} L^{3}\right)} 2 C_{F} e^{-\pi^{3 / 2} \sqrt{g_{s} N} T^{2} L^{2}} \\
& {\left[\cos \left(\frac{2 \pi m_{3}}{N}\right)+\cos \left(\frac{2 \pi n_{1}}{N}\right)+\cos \left(\frac{2 \pi n_{2}}{N}\right)\right] . }
\end{aligned}
$$


Turning on the twists in the $x_{1}-x_{2}, x_{1}-x_{3}$ and $x_{2}-x_{3}$ planes, corresponds in the supergravity theory to turning on expectations values for the $B^{N S}$ field along the appropriate two-cycle, eq.(24).

Note that in eq.(35) there is no dependence on $B^{R}$ because turning on an expectation value for $B^{R}$ field corresponds in the gauge theory to twisted boundary conditions in a plane involving $x_{0}$, the extra compactified direction, which has no intrinsic significance from the point of view of the three-dimensional theory.

\section{Non-supersymmetric Gauge Theory in Four Dimensions}

We turn next to discussing a non-supersymmetric four-dimensional theory. In analogy with our discussion of the previous section we will start with a five-dimensional theory realized by considering the world volume theory of $D 4$ branes and break supersymmetry by compactifying one of the brane directions on a circle with supersymmetry breaking boundary conditions [17, []. We compute the partition function of this theory as a function of the various electric and magnetic fluxes.

The supergravity solution is given by the near-horizon geometry of a configuration of $N$ near-extremal D4 branes [9]. The metric is

$$
d s^{2}=\left(\frac{r^{3 / 2}}{R^{3 / 2}}\right)\left(1-\frac{r_{H}^{3}}{r^{3}}\right) d x_{0}^{2}+\left(\frac{R^{3 / 2}}{r^{3 / 2}}\right) \frac{d r^{2}}{\left(1-\frac{r_{H}^{3}}{r^{3}}\right)}+\frac{r^{3 / 2}}{R^{3 / 2}}\left(d x_{1}^{2}+d x_{2}^{2}+d x_{3}^{2}+d x_{4}^{2}\right)+\sqrt{r R^{3}} d \Omega_{4}^{2},
$$

and the dilaton is

$$
\exp (\phi)=g_{s}\left(\frac{r}{R}\right)^{\frac{3}{4}},
$$

with $R^{3}=\pi g_{s} N\left(\alpha^{\prime}\right)^{3 / 2}$. From the point of view of the four-dimensional theory, $x_{0}$ is the extra spatial direction along which supersymmetry breaking boundary conditions are imposed, $x_{1}, x_{2}, x_{3}$ are the three spatial directions and $x_{4}$ is the Euclidean continuation of the time direction of the four-dimensional theory.

We are interested in the partition function of the gauge theory when the the three spatial and Euclidean time directions are each compactified on a circle of length $L$. There are six independent two cycles in this case and correspondingly six integers, modulo N, which characterize the twisted boundary conditions that can be introduced. Three of these directly correspond to the magnetic flux, while the remaining three, which correspond to twisted boundary conditions involving $x_{4}$, can be related to the electric flux by a Fourier transform.

By reasoning similar to that in $\S 2$ we find that turning on twisted boundary conditions along any of these six two-cycles corresponds to turning on an expectation value for $\int B^{N S}$, with the integral being evaluated along the two-cycle:

$$
\frac{2 \pi n_{I J}}{N}=\int_{\Sigma_{I J}} B^{N S}
$$


where $I, J$ go from $1, \ldots, 4$. The Supergravity Lagrangian contains a coupling of the form:

$$
\delta L=\int B^{N S} \wedge d C^{3} \wedge d C^{3}
$$

where $C^{3}$ denotes the 3 form RR field under which the $D 4$ brane is magnetically charged. This coupling is analogous to the Chern-Simons term in eq.(17) and ensures that the $\int B^{N S}$ can take only $N$ distinct values.

The leading dependence of the partition function on the discrete fluxes can now be determined. It is

$$
Z\left[n_{I J}\right]=e^{S_{B H}}\left[1+\tilde{C} e^{-\Lambda^{2} L^{2}} \sum_{I J} \cos \left(\frac{2 \pi n_{I J}}{N}\right)\right]
$$

The overall exponential factor arises from the free energy of the black hole geometry; an explicit calculation yields that $S_{B H} \sim N^{2}\left(g_{s} N \sqrt{\alpha^{\prime}} T\right) T^{4} L^{4}$. The second term arises from the one fundamental string instanton sector, the corresponding exponential factor comes from the minimal area of the world sheet which sweeps out the appropriate two-cycle. By considering large Wilson loops one can easily show that the theory confines. Reasoning entirely analogous to that in section 2.3 shows that the constant, $\Lambda^{2}$, is the string tension of the electric flux tube in the theory. Numerically,

$$
\Lambda^{2}=\frac{32}{27} \pi^{3}\left(g_{s} N \sqrt{\alpha^{\prime}} T\right) T^{2} .
$$

Finally, $\tilde{C}$ is the contribution of the determinant for fluctuations about the minimal area surface in the string path integral, it includes a factor proportional to $L^{2} T^{2}$ which arises from zero modes which correspond to moving the two-cycle in the two directions transverse to it. The equation Eq.(40), is the analogue of eq.(35) in the three-dimensional case. The dependence on the electric fluxes can be obtained by doing a Fourier transform in the $n_{I 4}$ variables.

A few comments are now in order. First, as in the finite temperature four-dimensional theory, the answer we obtain here has a simple interpretation in the gauge theory. The leading dependence on the discrete fluxes arises from electric flux tubes whose world volume sweeps out the relevant two-cycle of the torus. Second, eq.(40) is the leading contribution for a big torus, i.e., when $\Lambda L \gg 1$. Subleading terms arise from multiply wrapped and multi string configurations. Third, we have neglected both $\alpha^{\prime}$ corrections and $g_{s}$ corrections here. It is important to emphasize that neglecting the $\alpha^{\prime}$ corrections in particular requires us to work in a region of coupling constant space where the four-dimensional theory is much different from ordinary QCD. Extra Kaluza-Klein states in the theory have a mass of order the temperature $T$. The 't Hooft coupling of the four- dimensional theory at the cutoff scale, $T$, is $\lambda^{2}=g_{s} N \sqrt{\alpha^{\prime}} T$. Keeping the curvature small in string units requires, $\lambda^{2} \gg 1$. Thus the four-dimensional theory is already strongly coupled at the cutoff scale $\mathrm{F}$. Finally, from

\footnotetext{
$\ddagger$ Alternatively, we see from eq.(41) that when $\lambda^{2} \gg 1$, the string tension is bigger than the cutoff scale $T$.
} 
the point of view of the five-dimensional theory there can be additional twists along planes involving the temperature direction. The dependence on such twists arises due to D-2 branes whose world volume is a three cycle dual to the two-cycle along which the twisted boundary conditions are turned on. These twists do not have any intrinsic significance from the point of view of the four-dimensional theory.

The 4-torus has a geometric $S L(4, \mathbf{Z})$ duality symmetry and it is easy to verify that the answer obtained here, eq.(40), has that symmetry too once the discrete fluxes $n_{I J}$ are appropriately transformed. The significance of this duality symmetry was first realized by 't Hooft who formulated the conditions for invariance under it in terms of various duality relations. Of particular interest are $90^{\circ}$ rotations in the Euclidean theory, involving the time and one space directions, which exchange some of the electric and magnetic fluxes. The corresponding 't Hooft duality relations place strong constraints on the partition function which in turn constrain the phase structure of the gauge theory. It is worth sketching out how these general considerations apply in the present context. Let $Z[\vec{e}, \vec{m}]$ denote the partition function as a function of the electric and magnetic fluxes ( this is a discrete Fourier transform of eq.(40)) and let us consider it in the the infinite volume limit, $L \rightarrow \infty$. One can show that the duality relations allow for a solution which in this limit has the behavior: $Z[0, \vec{m}] / Z[0,0] \rightarrow 1$, while $Z[\vec{e}, \vec{m}] / Z[0,0] \rightarrow 0$. This implies that the free energy of a purely magnetic flux tube goes to zero in this limit whereas that of an electric flux tube diverges. Therefore, only electric flux tubes are stable and the theory is confining. In the large $N$ limit under discussion here, we know beforehand from a direct computation of the Wilson loop that the theory confines. Even so, it is revealing to explicitly compute the limiting behavior of $Z[0, \vec{m}] / Z[0,0]$ and $Z[\vec{e}, \vec{m}] / Z[0,0]$. One finds that it is of the form mentioned above, consistent with confinement.

We end with a brief discussion of another approximation to QCD, the strong coupling expansion in lattice gauge theory. The partition function as a function of the discrete fluxes can be easily computed in this approximation and goes as $Z\left[n_{I J}\right] \sim e^{-\Lambda^{2} A} \cos \left(\frac{2 \pi n_{L J}}{N}\right)$, where $\Lambda$ is the string tension. This is in agreement with eq.(40). Let us briefly review how this result is obtained. The fundamental degrees of freedom in lattice gauge theory are link variables. The action in terms of these is defined by taking the product of all the links around a plaquette. The leading contribution to the partition function which depends on the twisted boundary conditions then arises from tiling a two cycle of the torus minimally. This gives rise to the exponential dependence on the area. Once the two cycle is tiled the integrals over the interior link variables can be done simply using the fact that $\int d U U_{j}^{i}\left(U^{\dagger}\right)_{k}^{l}=\delta_{k}^{i} \delta_{j}^{l}$. Finally, the integrals over the edge link variables can be done after taking into account the twisted boundary conditions. Summing over both orientations for tiling gives the cosine dependence. In fact, intuitively, one would expect any confining theory to give rise to a dependence on the twisted boundary conditions of the form, eq.(40). Such a dependence can only arise from a non-local operator, the Wilson loop. This operator costs exponentially in the area in the confining theory and gives rise to the cosine term simply because it measure the flux by

\footnotetext{
${ }^{\S}$ Here we restrict ourselves to the large $\mathrm{N}$ limit, in general there is a finite $N$ correction to this.
} 
Stokes theorem. It is reassuring that our calculation above agrees with this expectation and with the strong coupling expansion.

\section{ACKNOWLEDGEMENTS}

We thank Bill Bardeen, Sumit Das, Sunil Mukhi, Ashoke Sen, and Spenta Wadia for useful discussions. A. D. would like to acknowledge the hospitality of the ICTP, the organizers of the Extended Workshop in String Theory, the Enrico Fermi Institute and the Particle Theory group at the University of Chicago where some of this work was completed. S. P. T. would like to thank the Theoretical Physics group at Fermilab for hospitality during his visit.

\section{References}

[1] O. Aharony, S. S. Gubser, J. Maldacena, H. Ooguri, and Y. Oz, "Large N Field Theories, String Theory, and Gravity," hep-th/9905111.

[2] O. Aharony and E. Witten, "Anti-de Sitter Space and the Center of The Gauge Group", hep-th/9611230, JHEP 9811:018,1998.

[3] M. J. Bowick, S. B. Giddings, J. A. Harvey, G. T. Horowitz and A. Strominger, Phys. Rev. Lett. 61 (1988) 2823.

[4] A. Brandhuber, N. Itzhaki, J. Sonnenschein, and S. Yankielowicz, "Wilson Loops in the Large-N Limit at Finite Temperature," Phys. Lett. B434 (1998) 36, hep-th/9803137.

[5] S. Coleman, J. Preskill, F. Wilczek, Nucl. Phys. B378 (1992) 175.

[6] S. S. Gubser, I. R. Klebanov, A. M. Polykov, "Gauge Theory Correlators from Noncrititcal String Theory," hep-th/9802109.

[7] D. J. Gross and H. Ooguri, "Aspects of Large $N$ Gauge Theory Dynamics as Seen by String Theory," hep-th/9805129.

[8] S. W. Hawking and D. Page, "Thermodynamics of Black Holes in Anti-de Sitter Space," Comm. Math. Phys., 87 (1988) 577.

[9] N. Itzhaki, J. Maldacena, J. Sonnenschein, and S. Yankielowicz, "Supergravity and the Large- $N$ Limit of Theories with Sixteen Supercharges," Phys. Rev. D58 (1998) 6004, hep-th/9802042.

[10] J. Maldacena, hep-th/9711200, Adv. Theor.Math.Phys.2:231-252,1998.

[11] J. Maldacena, "Wilson Loops in Large N Field Theories," hep/th9803002.

[12] J. Preskill, unpublished notes on quantum field theory. 
[13] S. J. Rey and J. Yee, "Macroscopic Strings as Heavy Quarks in Large N Gauge Theory and Anti-de Sitter Supergravity, hep-th/9803077.

[14] J. Schwarz, "An SL(2, Z) multiplet of Type-IIB Superstrings," Phys. Lett. B360 (1995) 13, hep-th/9508143.

[15] G. 't Hooft, "On the Phase Transition Towards Permanent Quark Confinement," Nucl. Phys. B138 (1978) 1; "A Property of Electric and Magnetic Flux in Nonabelian Gauge Theories," Nucl. Phys. B153 (1979) 141.

[16] E. Witten, "Anti-de Sitter Space and Holography," hep-th/9803002.

[17] E. Witten, "Anti-de Sitter Space, Thermal Phase Transition, And Confinement in Gauge Theories," hep-th/9803131, Adv. Theor.Math.Phys.2:505-532,1998.

[18] E. Witten, "AdS/CFT Correspondence And Topological Field Theory," hep-th/9812012, JHEP 9812:012,1998.

[19] E. Witten, "Bound States of Strings and p-Branes," Nucl. Phys. B460 (1996) 335, hep-th/9510135. 